\title{
Rapid Seedless Synthesis of Gold Nanoplates with Microscaled Edge Length in a High Yield and Their Application in SERS
}

\author{
Sheng Chen ${ }^{1,2} \cdot$ Pengyu Xu ${ }^{1,2} \cdot$ Yue $\mathrm{Li}^{1,2} \cdot$ Junfei Xue ${ }^{1,2} \cdot$ Song $\mathrm{Han}^{2} \cdot$ Weihui $\mathrm{Ou}^{2}$. \\ $\mathrm{Li} \mathbf{L i}^{1} \cdot$ Weihai $\mathrm{Ni}^{2}$
}

Received: 15 February 2016/ Accepted: 8 April 2016/Published online: 11 May 2016

(C) The Author(s) 2016. This article is published with open access at Springerlink.com

\begin{abstract}
We report a facile and reproducible approach toward rapid seedless synthesis of single crystalline gold nanoplates with edge length on the order of microns. The reaction is carried out by reducing gold ions with ascorbic acid in the presence of cetyltrimethylammonium bromide $(\mathrm{CTAB})$. Reaction temperature and molar ratio of CTAB/Au are critical for the formation of gold nanoplates in a high yield, which are, respectively, optimized to be $85{ }^{\circ} \mathrm{C}$ and 6 . The highest yield that can be achieved is $60 \%$ at the optimized condition. The synthesis to achieve the microscaled gold nanoplates can be finished in less than $1 \mathrm{~h}$ under proper reaction conditions. Therefore, the reported synthesis approach is a time- and costeffective one. The gold nanoplates were further employed as the surface-enhanced Raman scattering substrates and investigated individually. Interestingly, only those adsorbed with gold nanoparticles exhibit pronounced Raman signals of probe molecules, where a maximum enhancement factor of $1.7 \times 10^{7}$ was obtained. The obtained Raman enhancement can be ascribed to the plasmon coupling between the gold nanoplate and the nanoparticle adsorbed onto it.
\end{abstract}

Keywords Gold nanoplates · Seedless synthesis · SERS · CTAB

\section{Introduction}

Noble-metal nanocrystals have attracted a great amount of attention because of their unique light absorbing and scattering properties due to the localized surface plasmon resonance [1-3]. Wet chemical approaches have been developed toward the synthesis of a variety of metal nanocrystals $[4,5]$, such as nanospheres [6], nanorods $[7$,

Electronic supplementary material The online version of this article (doi:10.1007/s40820-016-0092-6) contains supplementary material, which is available to authorized users.

Weihai Ni

whni2012@sinano.ac.cn

1 Department of Chemistry, College of Sciences, Shanghai University, Shanghai 200444, People's Republic of China

2 Division of i-Lab \& Key Laboratory for Nano-Bio Interface Research, Suzhou Institute of Nano-Tech \& Nano-Bionics, Chinese Academy of Sciences, Suzhou 215123, Jiangsu, People's Republic of China
8], nanoplates $[9,10]$, nanowires $[11,12]$, etc. Compared to OD and 1D counterparts, 2D anisotropic nanocrystals, such as gold nanoplates, have large surface areas, sharp corners, and edges which can provide high enhancement of electric field [13-17], and therefore, achieve extensive applications including bio-imaging [18], nanodevices [19], surface-enhanced Raman scattering (SERS) [20], etc.

The growth of gold nanoplates can be directed by either templates or capping agents. By providing constrictions in a 2D space or dimension, planar substrates [14] and interfaces in lamellar bilayer membranes [21] have been used as effective templates for growth of gold nanoplates. Alternatively, capping agents can preferentially adsorb on a specific surface of gold so that the adsorption of gold ions to this surface is blocked, and the growth is restricted on a planar direction. These agents should be surfactants [22], polymers [23-26], biomolecules [27], and halide ions [28, 29]. Among them, cetyltrimethylammonium bromide (CTAB) is one of the most frequently used surfactants for the growth of gold nanoplates, which can be easily adsorbed onto the surface of gold through complexing with 
halide ions. For example, Mirkin group developed the seed-mediated growth of small gold nanoplates using CTAB as the capping agent [30]. Although the seed-mediated synthesis process effectively prohibits secondary nucleation and easily controls the size and shape of the final product, it involves multistep growth of seeds. To solve this problem, Huang group developed a seedless approach to synthesize gold nanoplates in the presence of CTAB via thermal reduction, where reaction solutions were preheated before they were mixed together to ensure the control of the size distribution [22, 31]. However, long preheating time will result in higher time consumption for the process and prove to be cost ineffective. Recently, high-yield synthesis of gold nanoplates with submicron edge length was reported where iodide ions were used as both the capping and etching agents [28]. However, rapid synthesis of gold nanoplates in microscaled edge length with high yield, simplicity, and low-cost still remains challenging.

Herein, we report a facile and reproducible approach of rapid, seedless synthesis of single crystalline gold nanoplates with edge lengths in micron orders of magnitude. The reaction was carried out by reducing gold ions with ascorbic acid in the presence of CTAB. The reaction temperature and molar ratio of $\mathrm{CTAB} / \mathrm{Au}$ on the products were examined in detail. The SERS properties of the assynthesized gold nanoplates were also investigated.

\section{Experimental}

\subsection{Chemicals}

Hydrogen tetrachloroaurate tetrahydrate $\left(\mathrm{HAuCl}_{4} \cdot 3 \mathrm{H}_{2} \mathrm{O}\right)$, L-ascorbic acid (AA), cetyltrimethylammonium bromide (CTAB), and 4-Mercaptophenol (Mph) were purchased from Sigma-Aldrich. All chemicals and reagents were used without any further purification. Ultrapure water was obtained from the Milli-Q system (18.2 $\mathrm{M} \Omega \mathrm{cm}^{-1}$ ).

\subsection{Synthesis of Gold Nanoplates}

A typical synthesis procedure is as follows: first, $100 \mu \mathrm{L}$ of $0.1 \mathrm{M} \mathrm{HAuCl}_{4}$ was added into $3 \mathrm{~mL}$ of $0.02 \mathrm{M} \mathrm{CTAB}$ aqueous solution in a plastic tube, and the mixture was left undisturbed for several minutes. Then, $100 \mu \mathrm{L}$ of $0.1 \mathrm{M}$ AA was added to the mixture, followed by rapid inversion for $10 \mathrm{~s}$. The resultant solution was immediately placed in a water bath of $85^{\circ} \mathrm{C}$ and kept undisturbed for about $1 \mathrm{~h}$. The products were washed by centrifugation at $4000 \mathrm{rpm}$ for $10 \mathrm{~min}$ and finally dispersed in deionized water.

\subsection{Preparation of SERS Substrates}

The SERS substrate was prepared as follows: gold nanoplate solution was drop-casted onto a clean silicon substrate. The substrate was rinsed and blown dry by nitrogen gas. Afterward, it was immersed into a solution of gold nanoparticles for several minutes, allowing deposition of gold nanoparticles on the gold nanoplates. After thoroughly rinsing with water for several times, it was immersed into a solution of $0.01 \mathrm{M} \mathrm{Mph}$ for $3 \mathrm{~h}$. The substrate was carefully rinsed and blown dry by nitrogen gas before the SERS measurement. A cross-bar was finally marked on the substrate for locating the gold nanoplate and investigating them individually under the optical microscope and SEM.

\subsection{Characterizations}

The extinction spectra of the gold nanoplates were recorded using Agilent Cary $60 \mathrm{UV}-\mathrm{Vis}$ spectrophotometer using a cuvette having $0.5-\mathrm{cm}$ path length. The morphology of the gold nanoplates was characterized by Hitachi S-4800 field emission and FEI Quanta 250 FEG SEMs. Powder X-ray diffraction (XRD) patterns were recorded on a Bruker D8 Advance powder X-ray diffractometer at a scanning rate of $4^{\circ} \mathrm{min}^{-1}$, using $\mathrm{Cu}-\mathrm{K} \alpha$ radiation $(\lambda=1.54056 \AA)$. Raman scattering spectra were measured on a micro-Raman system (HR evolution 260, Horiba). The sample was excited at $633 \mathrm{~nm}$ and 4 $\mathrm{mW}$ in the Raman measurement. The Raman scattered light was collected using an Olympus objective $(100 X$, N.A. $=0.9$, W.D. $=1 \mathrm{~mm})$. Raman spectra were recorded using a grating of 600 lines per $\mathrm{mm}$ with an integration time of $15 \mathrm{~s}$.

\section{Results and Discussion}

As shown in Fig. 1, the synthesis of the gold nanoplates is very straightforward. To be specific, aqueous solutions of $\mathrm{HAuCl}_{4}$ and CTAB were first mixed and left undisturbed at

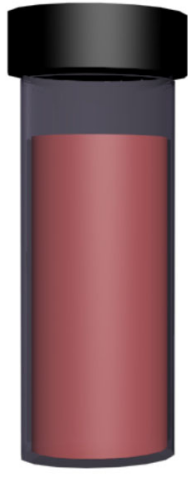

$\mathrm{Au}^{3+} / \mathrm{CTAB}$

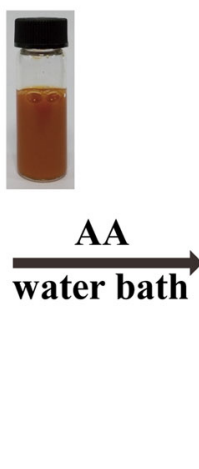

Au nanoplates
Fig. 1 Schematic illustration of the synthesis of the gold nanoplates 
room temperature for several minutes. The color of the mixture solution slowly changed to red brown owing to the formation of $\mathrm{AuBr}_{4}^{-}$complex ions [25, 32, 33]. Afterward, a reducing reagent of AA was injected, which quickly makes the mixture to be colorless. The reaction solution was kept undisturbed in a water bath of $85{ }^{\circ} \mathrm{C}$ for $1 \mathrm{~h}$, and some precipitation of the resultant products was found at the bottom of the glass vial.

Figure 2a shows the extinction spectra of the as-prepared gold nanoplates in an aqueous solution, and the inset shows the comparison of the colors of the solution before and after the synthesis reaction. The gold nanoplates exhibit a broad absorption band starting from $500 \mathrm{~nm}$, which can be ascribed to the dipole and quadrupole plasmon resonances [25, 34, 35]. The morphologies of resultant products were examined by SEM (see Fig. $2 \mathrm{~b}$ ) and statistically analyzed, and the results are shown in Fig. S1 of Supporting Information (SI). Although a small amount of spherical nanoparticles were observed as well, the resultant plate-like products were mainly composed of $8.5 \%$ triangular and $91.5 \%$ equilateral hexagonal nanoplates with average edge lengths of $3.5 \mu \mathrm{m}$, and thicknesses of around $114 \mathrm{~nm}$ (obtained from the tilted SEM image shown in the inset), which strongly confirms the relatively monodisperse and uniform nanoplates.

The XRD pattern of gold nanoplates was recorded using a quartz substrate (Fig. 3). The diffraction peaks are assigned to (111), (200), (220), and (311) planes of facecentered-cubic structure of Au (PDF No. 04-0784). Note that the intensity of $\mathrm{Au}$ (111) peak is much stronger than those of (200), (220), and (311). The diffraction intensity ratio of $(200) /(111)$ is extremely lower than the standard file ( 0.0051 versus 0.52$)$. This XRD result clearly indicates that the as-synthesized gold naonoplates are single crystalline and possess a preferred plane of (111).

To achieve a high yield, the synthesis conditions that affect the growth of gold nanoplates were finely optimized.

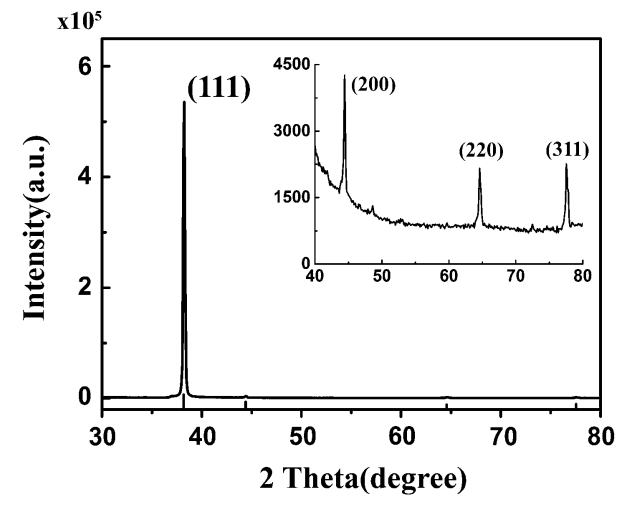

Fig. 3 XRD pattern of the as-prepared gold nanoplates deposited on quartz

It is known that the reaction temperature plays an important role in the formation of anisotropic nanostructures [36, 37]. Therefore, in our experiment, the reaction temperature was varied from 25 to $95{ }^{\circ} \mathrm{C}$, as illustrated in Fig. 4. Figure 4a shows the morphologies of resultant products synthesized at room temperature $\left(25^{\circ} \mathrm{C}\right)$ where most of them are identified as plate- and sphere-like particles. Both the yields and the edge lengths of the nanoplates start to increase when the temperature is increased. Figure $4 b, c$ shows the products synthesized at 45 and $65^{\circ} \mathrm{C}$, respectively. At $85^{\circ} \mathrm{C}$, a large amount of hexagonal nanoplates with edge length of about $\sim 3.5 \mu \mathrm{m}$ can be found where the highest yield of $60 \%$ is obtained (Fig. 2b). The extinction spectrum suggests that increasing the reaction temperature further to $95^{\circ} \mathrm{C}$ leads to both inhomogeneous size distribution of the gold nanoplates and the decrease in the yield to $53 \%$ (Fig. 4d). These results indicate that the most preferable reaction temperature for the high-yield synthesis of the gold nanoplates is $85{ }^{\circ} \mathrm{C}$. Extinction spectra of the $\mathrm{Au}$ products grown at different temperature are shown in Fig. S1, Supporting Information (SI). In contrast to the turbid appearance of the solutions obtained at 25 and
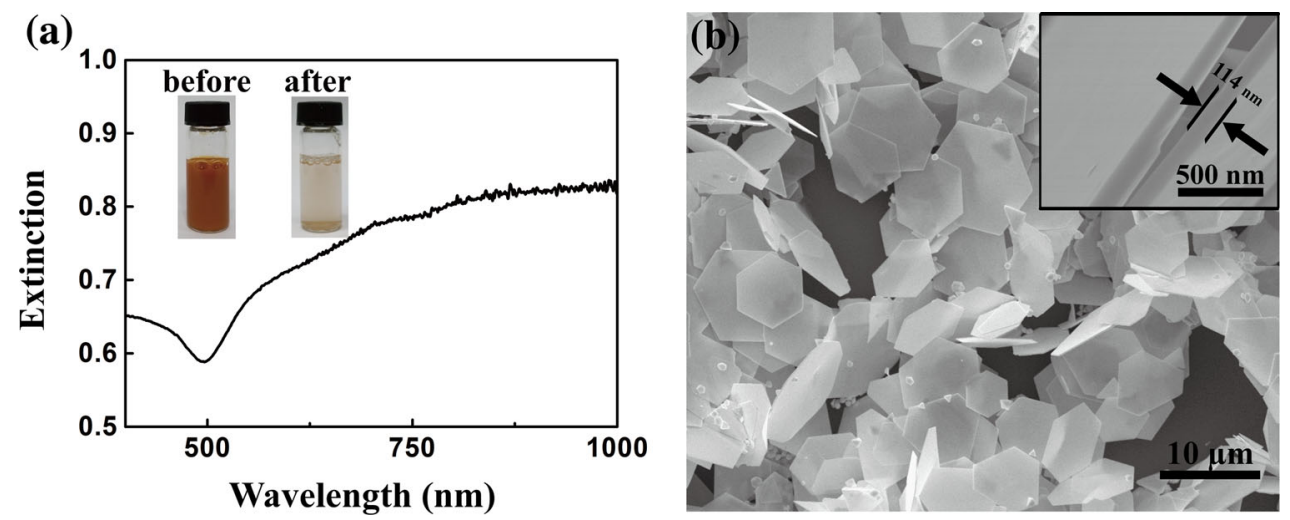

Fig. 2 a The extinction spectrum of gold nanoplates in aqueous solution. The inset shows the photographs of the solutions before and after the growth of gold nanoplates. b The SEM images of gold nanoplates. The inset shows a cross-sectional SEM image of the gold nanoplates 

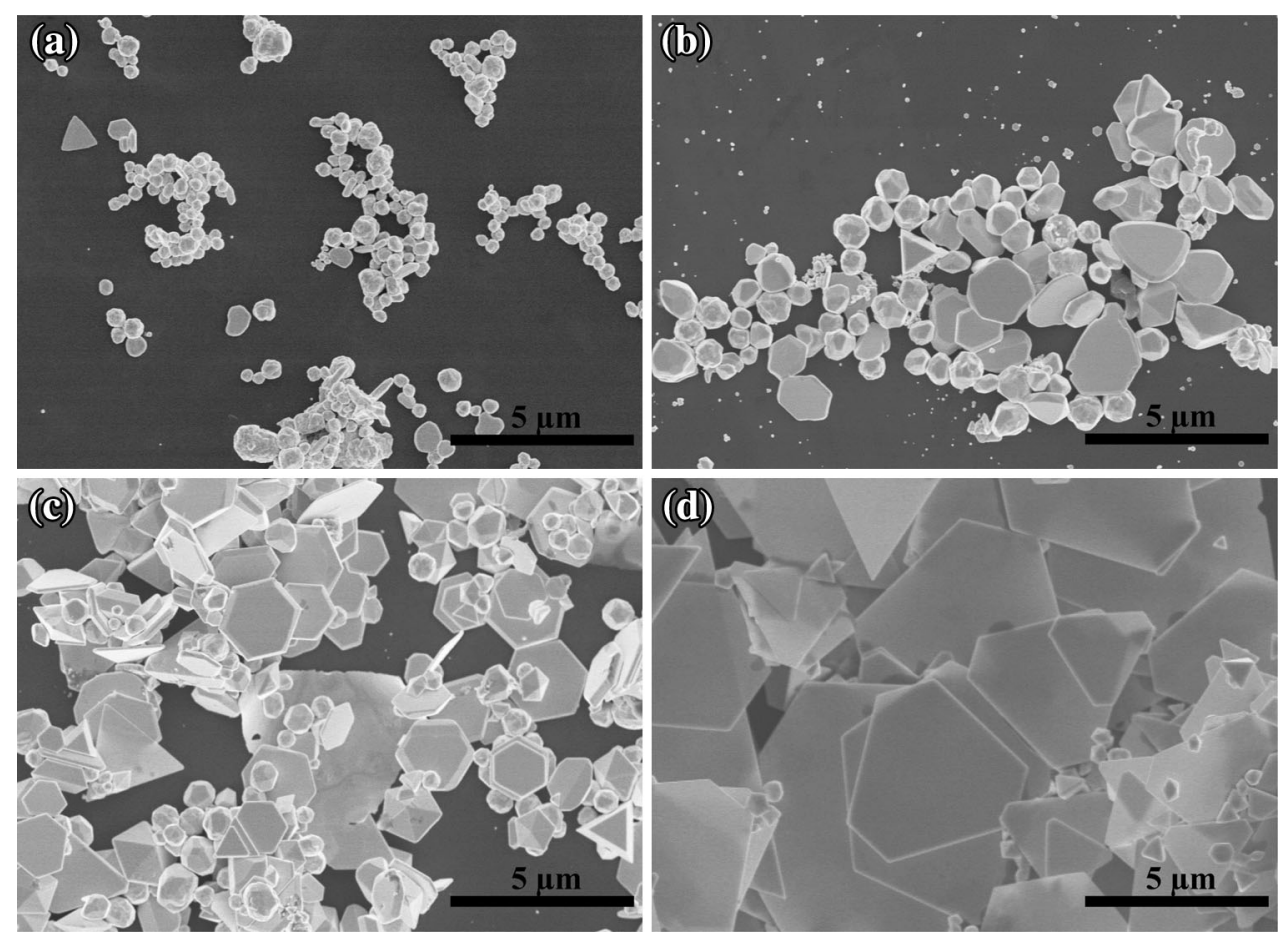

Fig. 4 SEM images of the samples prepared at a $25{ }^{\circ} \mathrm{C}$, b $45{ }^{\circ} \mathrm{C}, \mathbf{c} 65^{\circ} \mathrm{C}$, and $\mathbf{d} 95{ }^{\circ} \mathrm{C}$. The molar ratio of $\mathrm{Br}^{-} / \mathrm{Au}$ is fixed at $6: 1$

$45^{\circ} \mathrm{C}$, those at 65,85 , and $95^{\circ} \mathrm{C}$ exhibit features of plasmon resonances belonging to the gold nanoplates, which is consistent with the SEM images. The gold nanoplate solution obtained at $85^{\circ} \mathrm{C}$ shows the highest extinction intensity among the these three, suggesting the highest yield.

Besides the reaction temperature, the molar ratio of $\mathrm{Br}^{-} /$ $\mathrm{Au}$ has greatly significant influence on the formation of the gold nanoplates. Figure 5 shows the gold nanoplates synthesized at various $\mathrm{Br}^{-} / \mathrm{Au}$ ratios with the addition of different volumes of $0.2 \mathrm{M} \mathrm{NaBr}$ to the reaction solution. The synthesis was carried out in a water bath of $85^{\circ} \mathrm{C}$ for $1 \mathrm{~h}$. As shown in Fig. 5a, when molar ratio of $\mathrm{Br}^{-} / \mathrm{Au}$ is $1: 1$, the final products are almost nanoparticles, and no plate-like nanocrystals were found. Nanoplates start to appear in the resultant products when molar ratio of $\mathrm{Br}^{-} /$ $\mathrm{Au}$ is increased to 3:1 (Fig. 5b). When molar ratio of $\mathrm{Br}^{-} /$ $\mathrm{Au}$ is tuned to $6: 1$, nanoplates dominate in the resultant products (Fig. 2b). The amount of nanoplates start to decrease when molar ratio of $\mathrm{Br}^{-} / \mathrm{Au}$ is further increased (Fig. 5c, d). These results indicate that the $\mathrm{Br}^{-} / \mathrm{Au}$ molar ratio of $6: 1$ is the most preferable for the formation of nanoplates. Also, $\mathrm{CTA}^{+}$as the capping agent is necessary in the synthesis, in which a proper concentration of $20 \mathrm{mM}$ was used. The results were also confirmed by the extinction spectra (Fig. S1b). A prominent plasmon peak at around $550 \mathrm{~nm}$ can be found for the solutions obtained at $\mathrm{Br}^{-} / \mathrm{Au}$ ratios of $1: 1$ and 3:1, indicating the presence of considerable amounts of spherical gold nanoparticles in the solutions. This peak becomes less observable at $\mathrm{Br}^{-} / \mathrm{Au}$ ratios of $6: 1$, and the spectrum in the near infrared increases, suggesting high yield of nanoplates. The extinction intensity drops when the ratio is further increased to $12: 1$ and 30:1.

It is generally believed that the formation pathway of nanoplates is "kinetically controlled" along with the surfactant as capping agent or template-like CTAB and PVP $[16,33]$. On the basis of the experimental evidence, the reaction temperature of $85{ }^{\circ} \mathrm{C}$ and the $\mathrm{Br}^{-} / \mathrm{Au}$ molar ratio of 6:1 are optimal conditions for obtaining high-yield gold nanoplates. To investigate this reaction route, a series of experimental investigations on the formation process were carried out through sampling gold nanoplates with the increasing reaction times. Figure 6 shows the sizes and shapes of gold nanoplates under different increasing reaction times. It can be found that small plate-like nuclei are formed in less than $5 \mathrm{~min}$ (Fig. 6a). They grow bigger into nanoplates with micrometer edge length within $30 \mathrm{~min}$ (Fig. 6b), and a high yield is obtained after $60 \mathrm{~min}$ (Fig. 6c). The experiment was performed at the preferable reaction temperature and concentration of $\mathrm{Br}^{-}$and $\mathrm{CTA}^{+}$. This result indicates that under the preferable reaction condition, a minimum 1-h reaction time is essential for the synthesis of gold nanoplates in a high yield.

Recently, many endeavors have been devoted to the fabrication of efficient SERS substrates. For example, the 

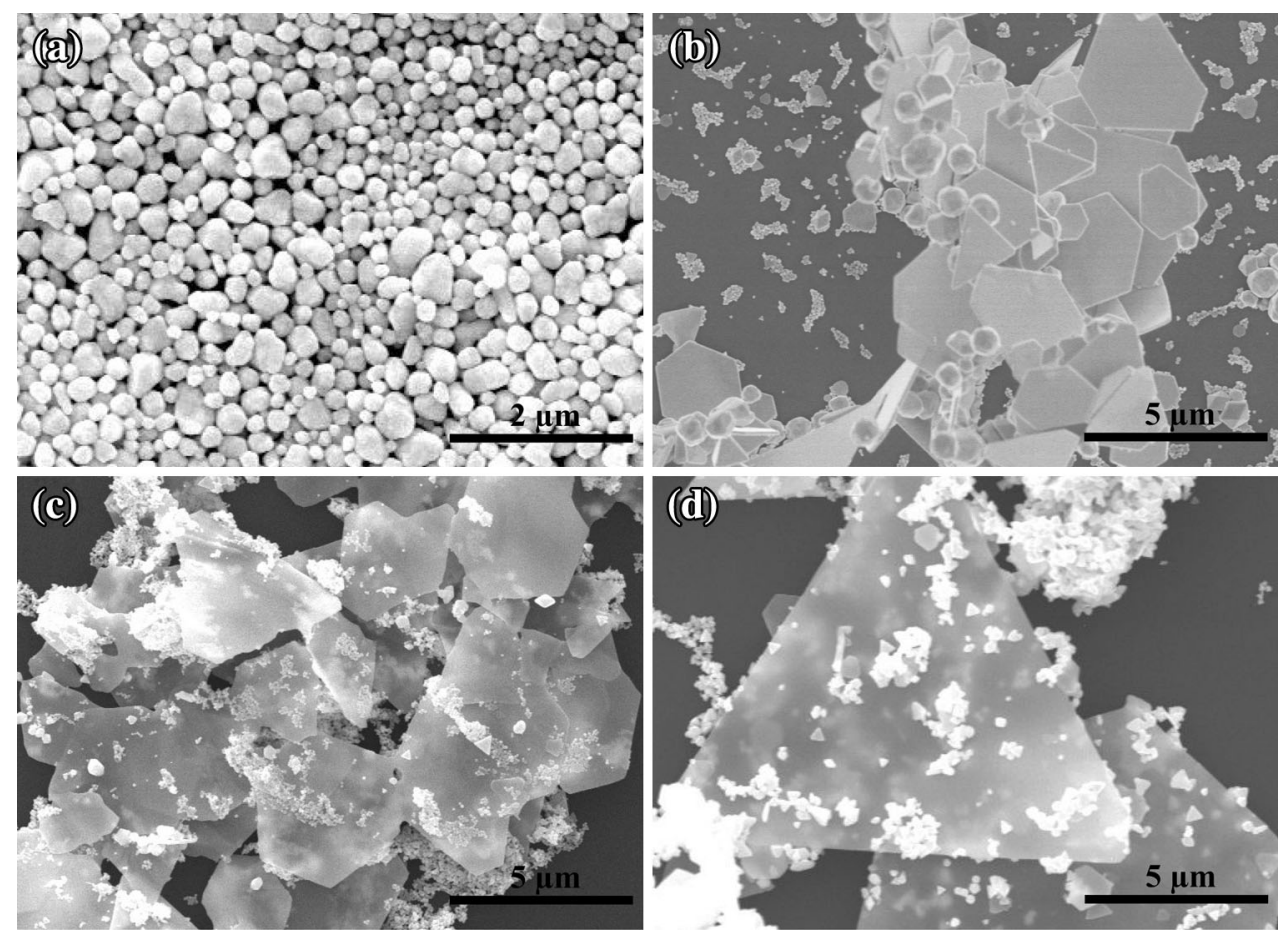

Fig. 5 SEM images of the samples prepared with different molar ratios of $\mathrm{Br}^{-} / \mathrm{Au}: \mathbf{a} 1: 1, \mathbf{b} 3: 1, \mathbf{c}$ 12:1, and $\mathbf{d}$ 30:1. The reaction temperature is fixed at $85{ }^{\circ} \mathrm{C}$

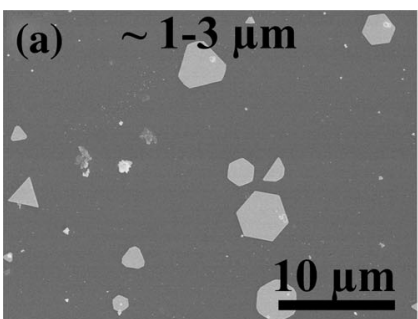

5 min

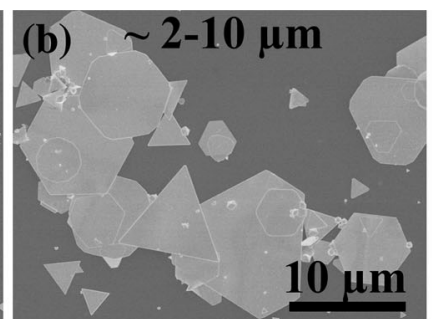

$30 \mathrm{~min}$

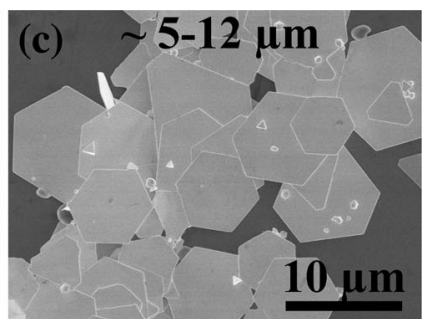

$60 \mathrm{~min}$

Fig. 6 SEM images of the gold nanoplates obtained a less than $5 \mathrm{~min}$, b within $30 \mathrm{~min}$, and $\mathbf{c}$ after $60 \mathrm{~min}$

nanoscaled gaps provided by sandwiched structures can greatly augment the Raman signals [38-42]. Individual gold nanoplates, however, are rarely used as SERS substrates alone due to their flat surface. Here, we demonstrate that the gold nanoplates with adsorbed gold nanoparticles can be used as efficient SERS substrates for achieving high enhancement factors (EFs). The Raman measurements were performed under excitation of $633 \mathrm{~nm}$ where $\mathrm{Mph}$ was used as the probing molecule. The same objective was used for the excitation and collection of the Raman scattering light. Figure $7 \mathrm{a}, \mathrm{b}$ shows a typical gold nanoplate with adsorbed gold nanoparticle. Under the optical microscope, the same nanoplate was located and positioned in the center of the optical view (Fig. 7c). The focal point of the excitation laser at $633 \mathrm{~nm}$ was thereafter positioned exactly on the nanoparticle (Fig. 7d). Raman responses from gold nanoplates with and without adsorbed nanoparticles are measured and compared (Fig. 8a). Raman spectra from the individual corresponding gold nanoplates are shown in Fig. 8b. The Raman signals from nanoplates without nanoparticles adsorbed are so weak that no peaks can be identified on the spectrum (cases 1 and 2). It is also the case when a nanoparticle is adsorbed onto the edge of the nanoplate (case 3). This result is related to the lack of hot spots no matter whatever the shapes of these nanoplates be. Interestingly, strong Raman signals are observable when some nanoparticles are adsorbed on the upper surface of the nanoplates (cases 4, 5, and 6), and the excitation laser beam is focused on the nanoparticles. In such cases, hot spots are formed in the gap between nanoparticles and nanoplates, which greatly enhances the Raman response because of the interparticle electromagnetic coupling [4345]. Characteristic Raman peaks of Mph can be found on the measured spectra at $830,1014,1083,1175$, and 

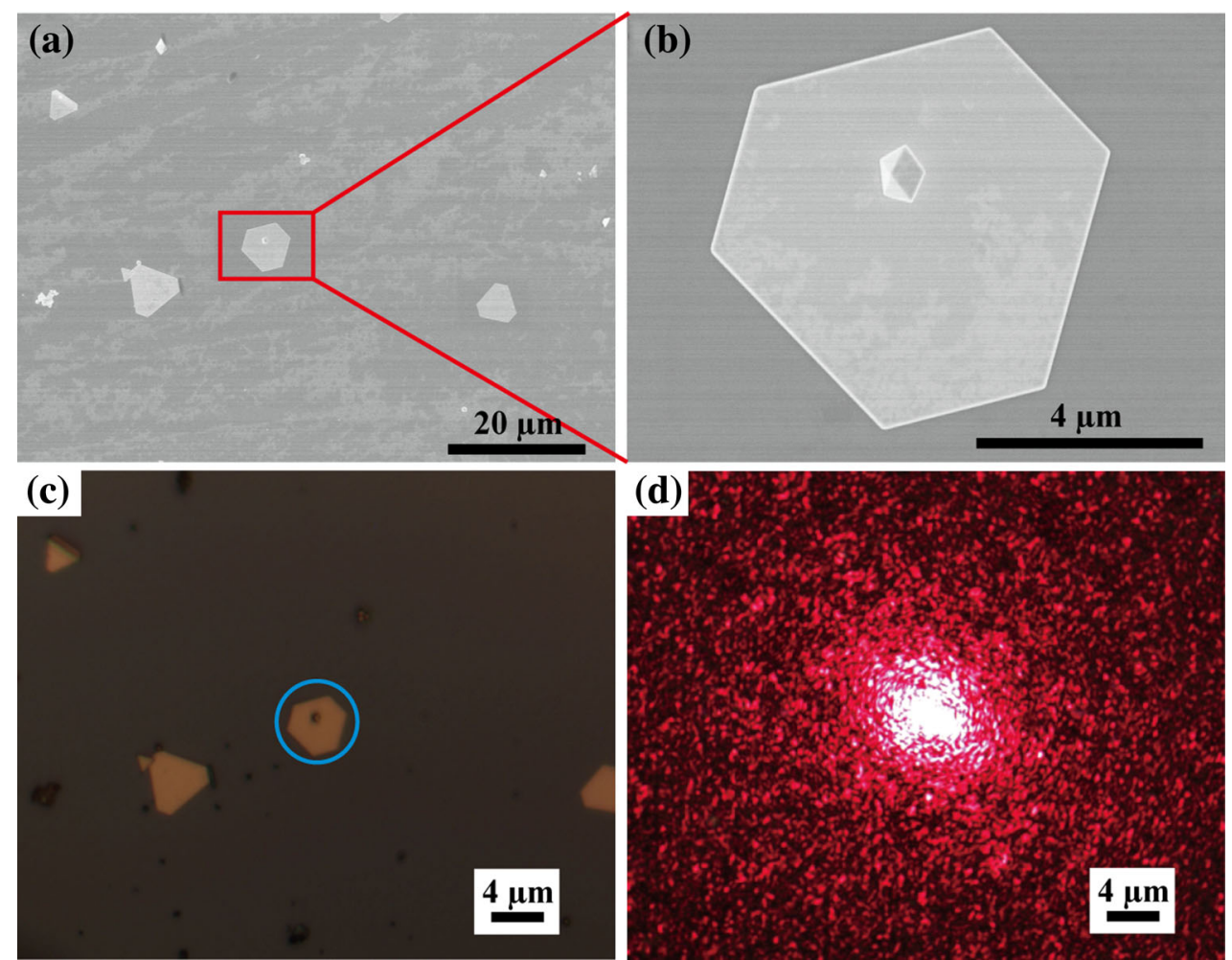

Fig. 7 a The SEM images of gold nanoplates with a nanoparticle adsorbed. b Zoomed-in SEM image of (a). c Corresponding optical image with the nanoplate highlighted in the blue circle. $\mathbf{d}$ The focal point of the excitation laser positioned on the nanoparticle. (Color figure online)

(a)

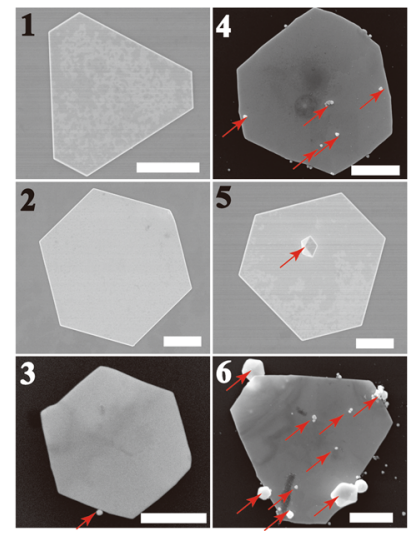

(b)

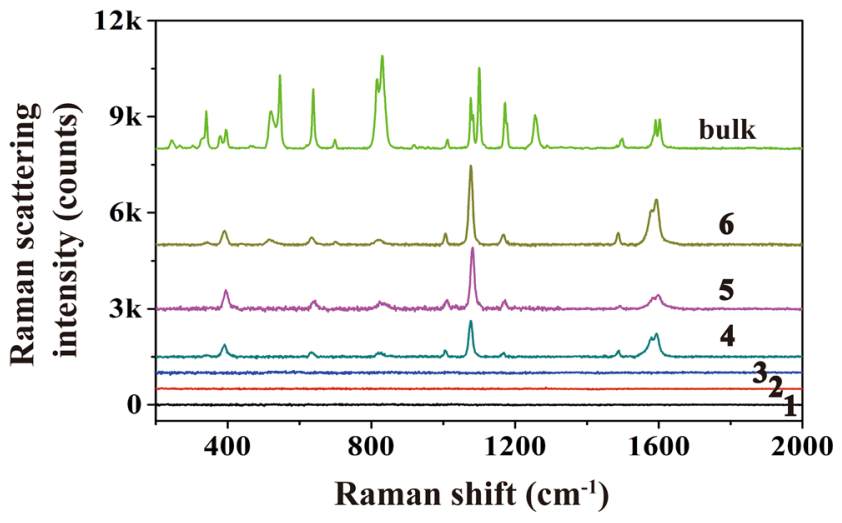

Fig. 8 a SEM images of gold nanoplates without any adsorbed nanoparticles (cases 1 and 2), those with adsorbed nanoparticles on the side (case 3 ), and those with the adsorbed nanoparticles on the surface (cases 4, 5, and 6). The nanoparticles are indicated by red arrows. The scale bars are $2 \mu \mathrm{m}$. b Raman spectra of bulk MPh and corresponding nanoplates in cases from 1 to 6 . (Color figure online)

$1600 \mathrm{~cm}^{-1}$. Among these peaks 830,1014, and $1083 \mathrm{~cm}^{-1}$ are, respectively, assigned to $\mathrm{C}-\mathrm{H}$ wagging, $\mathrm{C}-\mathrm{C}$ bending, and $\mathrm{C}-\mathrm{S}$ stretching, and 1175 and $1600 \mathrm{~cm}^{-1}$ are belong to C-H bending modes [43, 46, 47]. Note that the Raman spectra from the SERS substrates are slightly different from the bulk (Fig. 8b).

Enhancement factors (EFs) of the samples are evaluated by the equation of $\mathrm{EF}=($ ISERS/IBulk) $(\mathrm{NBulk} / \mathrm{NSERS})$, where ISERS and IBulk represent the Raman intensity values measured on the SERS substrate and bulk Mph, respectively. NSERS and NBulk are the numbers of Mph molecules adsorbed on the SERS and bulk samples inside of the laser spot, respectively. The EFs of SERS peaks at $1011,1081,1492$, and $1599 \mathrm{~cm}^{-1}$ are evaluated to be $1.5 \times 107,4.0 \times 106,1.7 \times 107$, and $8.1 \times 106$, respectively. These results suggest that the gold nanoplates can be 
used as ideal SERS substrates for detecting Raman analytes.

\section{Conclusions}

In summary, we successfully developed a simple but effective route to the synthesis of single crystalline gold nanoplates with edge length on the order of microns. Optimized reaction temperature and molar ratio of CTAB/ $\mathrm{Au}$ are found to be, respectively, $85^{\circ} \mathrm{C}$ and $6: 1$ for the formation of gold nanoplates in a high yield of $60 \%$. The synthesis to achieve the microscaled gold nanoplates can be finished in less than $1 \mathrm{~h}$ under proper reaction conditions. Therefore, the reported synthesis is time- and cost effective. The gold nanoplates were further employed as the SERS substrates and investigated individually. Interestingly, only those adsorbed with gold nanoparticles exhibit pronounced Raman signals of probe molecules, where a maximum enhancement factor of $1.7 \times 10^{7}$ was obtained. Our work demonstrated that a designed nanostructure consisting of a nanoplate adsorbed with a nanoparticle on its upper surface can be used as an efficient SERS substrate for reproducible enhancement.

Acknowledgments This work is supported by the National Natural Science Foundation of China (NSFC) (Grants 21271181 and 21473240), Ministry of Science and Technology of China (Intergovernmental S\&T Cooperation Project, Grant No. 6-10), and the Thousand Youth Talents Program of China.

Open Access This article is distributed under the terms of the Creative Commons Attribution 4.0 International License (http://crea tivecommons.org/licenses/by/4.0/), which permits unrestricted use, distribution, and reproduction in any medium, provided you give appropriate credit to the original author(s) and the source, provide a link to the Creative Commons license, and indicate if changes were made.

\section{References}

1. M.A. El-Sayed, Some interesting properties of metals confined in time and nanometer space of different shapes. Acc. Chem. Res. 34(4), 257-264 (2001). doi:10.1021/ar960016n

2. P. Zijlstra, M. Orrit, Single metal nanoparticles: optical detection, spectroscopy and applications. Rep. Prog. Phys. 74(10), 106401 (2011). doi:10.1088/0034-4885/74/10/106401

3. W. Ni, X. Kou, Z. Yang, J. Wang, Tailoring longitudinal surface plasmon wavelengths, scattering and absorption cross sections of gold nanorods. ACS Nano 2(4), 677-686 (2008). doi:10.1021/ nn7003603

4. K.M. Koczkur, S. Mourdikoudis, L. Polavarapu, S.E. Skrabalak, Polyvinylpyrrolidone (PVP) in nanoparticle synthesis. Dalton Trans. 44(41), 17883-17905 (2015). doi:10.1039/c5dt02964c

5. L. Polavarapu, S. Mourdikoudis, I. Pastoriza-Santos, J. PerezJuste, Nanocrystal engineering of noble metals and metal chalcogenides: controlling the morphology, composition and crystallinity. CrystEngComm 17(20), 3727-3762 (2015). doi:10. 1039/c5ce00112a

6. Q. Ruan, L. Shao, Y. Shu, J. Wang, H. Wu, Growth of monodisperse gold nanospheres with diameters from 20 to $220 \mathrm{~nm}$ and their core/satellite nanostructures. Adv. Opt. Mater. 2(1), 65-73 (2014). doi:10.1002/adom.201300359

7. T.K. Sau, C.J. Murphy, Room temperature, high-yield synthesis of multiple shapes of gold nanoparticles in aqueous solution. J. Am. Chem. Soc. 126(28), 8648-8649 (2004). doi:10.1021/ ja047846d

8. T.K. Sau, C.J. Murphy, Seeded high yield synthesis of short au nanorods in aqueous solution. Langmuir 20(15), 6414-6420 (2004). doi:10.1021/la049463z

9. Y. Shao, Y. Jin, S. Dong, Synthesis of gold nanoplates by aspartate reduction of gold chloride. Chem. Commun. 9, 1104-1105 (2004). doi:10.1039/b315732f

10. T. Soejima, N. Kimizuka, One-pot room-temperature synthesis of single-crystalline gold nanocorolla in water. J. Am. Chem. Soc. 131(40), 14407-14412 (2009). doi:10.1021/ja904910m

11. Z. Huo, C.K. Tsung, W. Huang, X. Zhang, P. Yang, Sub-two nanometer single crystal Au nanowires. Nano Lett. 8(7), 2041-2044 (2008). doi:10.1021/n18013549

12. F. Kim, K. Sohn, J. Wu, J. Huang, Chemical synthesis of gold nanowires in acidic solutions. J. Am. Chem. Soc. 130(44), 14442-14443 (2008). doi:10.1021/ja806759v

13. B. Radha, G.U. Kulkarni, A real time microscopy study of the growth of giant $\mathrm{Au}$ microplates. Cryst. Growth Des. 11(1), 320-327 (2011). doi:10.1021/cg1015548

14. X. Wu, R. Kullock, E. Krauss, B. Hecht, Single-crystalline gold microplates grown on substrates by solution-phase synthesis. Cryst. Res. Technol. 50(8), 595-602 (2015). doi:10.1002/crat. 201400429

15. J. Huang, L. Lin, D. Sun, H. Chen, D. Yang, Q. Li, Bio-inspired synthesis of metal nanomaterials and applications. Chem. Soc. Rev. 44(17), 6330-6374 (2015). doi:10.1039/c5cs00133a

16. H. Hu, J.Y. Zhou, Q.S. Kong, C.X. Li, Two-dimensional au nanocrystals: shape/size controlling synthesis, morphologies, and applications. Part. Part. Syst. Char. 32(8), 796-808 (2015). doi:10.1002/ppsc. 201500035

17. Ping Cai, Shu-Mei Zhou, De-Kun Ma, Shen-Nan Liu, Wei Chen, Shao-Ming Huang, $\mathrm{Fe}_{2} \mathrm{O}_{3}$-modified porous $\mathrm{BiVO}_{4}$ nanoplates with enhanced photocatalytic activity. Nano-Micro Lett. 7(2), 183-193 (2015). doi:10.1007/s40820-015-0033-9

18. N. Li, P.X. Zhao, D. Astruc, Anisotropic gold nanoparticles: synthesis, properties, applications, and toxicity. Angew. Chem. Int. Ed. 53(7), 1756-1789 (2014). doi:10.1002/anie. 201300441

19. J.S. Huang, V. Callegari, P. Geisler, C. Bruning, J. Kern et al., Atomically flat single-crystalline gold nanostructures for plasmonic nanocircuitry. Nat. Commun. 1(9), 749-763 (2010). doi:10.1038/ncomms 1143

20. T. Deckert-Gaudig, V. Deckert, Ultraflat transparent gold nanoplates: ideal substrates for tip-enhanced Raman scattering experiments. Small 5(4), 432-436 (2009). doi:10.1002/smll. 200801237

21. J. Niu, D. Wang, H. Qin, X. Xiong, P. Tan et al., Novel polymerfree iridescent lamellar hydrogel for two-dimensional confined growth of ultrathin gold membranes. Nat. Commun. 5, 3313 (2014). doi:10.1038/ncomms4313

22. H.C. Chu, C.H. Kuo, M.H. Huang, Thermal aqueous solution approach for the synthesis of triangular and hexagonal gold nanoplates with three different size ranges. Inorg. Chem. 45(2), 808-813 (2006). doi:10.1021/ic051758s

23. B. Wiley, Y. Sun, B. Mayers, Y. Xia, Shape-controlled synthesis of metal nanostructures: the case of silver. Chemistry 11(2), 454-463 (2005). doi:10.1002/chem.200400927 
24. Y. Xiong, I. Washio, J. Chen, H. Cai, Z.Y. Li, Y. Xia, Poly(vinyl pyrrolidone): a dual functional reductant and stabilizer for the facile synthesis of noble metal nanoplates in aqueous solutions. Langmuir 22(20), 8563-8570 (2006). doi:10.1021/la061323x

25. C. Wang, C. Kan, J. Zhu, X. Zeng, X. Wang, H. Li, D. Shi, Synthesis of high-yield gold nanoplates: fast growth assistant with binary surfactants. J. Nanomater. 2010, 1-9 (2010). doi:10. $1155 / 2010 / 969030$

26. W. Zhu, Y.Y. Wu, C.Y. Wang, M. Zhang, G.X. Dong, Fabrication of large-area 3-d ordered silver-coated colloidal crystals and macroporous silver films using polystyrene templates. NanoMicro Lett. 5(3), 182-190 (2013). doi:10.5101/nml.v5i3.082-190

27. B. Liu, J. Xie, J.Y. Lee, Y.P. Ting, J.P. Chen, Optimization of high-yield biological synthesis of single-crystalline gold nanoplates. J. Phys. Chem. B 109(32), 15256-15263 (2005). doi:10. 1021/jp051449n

28. L. Chen, F. Ji, Y. Xu, L. He, Y. Mi, F. Bao, B. Sun, X. Zhang, Q. Zhang, High-yield seedless synthesis of triangular gold nanoplates through oxidative etching. Nano Lett. 14(12), 7201-7206 (2014). doi:10.1021/n1504126u

29. Y. Huang, A.R. Ferhan, Y. Gao, A. Dandapat, D.H. Kim, Highyield synthesis of triangular gold nanoplates with improved shape uniformity, tunable edge length and thickness. Nanoscale 6(12), 6496-6500 (2014). doi:10.1039/c4nr00834k

30. J.E. Millstone, G.S. Métraux, C.A. Mirkin, Controlling the edge length of gold nanoprisms via a seed-mediated approach. Adv. Funct. Mater. 16(9), 1209-1214 (2006). doi:10.1002/adfm. 200600066

31. W.L. Huang, C.H. Chen, M.H. Huang, Investigation of the growth process of gold nanoplates formed by thermal aqueous solution approach and the synthesis of ultra-small gold nanoplates. J. Phys. Chem. C 111(6), 2533-2538 (2007). doi:10.1021/ jp0672454

32. L. Wang, X. Chen, J. Zhan, Y. Chai, C. Yang, L. Xu, W. Zhuang, B. Jing, Synthesis of gold nano- and microplates in hexagonal liquid crystals. J. Phys. Chem. B 109(8), 3189-3194 (2005). doi:10.1021/jp0449152

33. S. Hong, J.A.I. Acapulco, H.-J. Jang, A.S. Kulkarni, S. Park, Kinetically controlled growth of gold nanoplates and nanorods via a one-step seed-mediated method. Bull. Korean Chem. Soc. 35(6), 1737-1742 (2014). doi:10.5012/bkcs.2014.35.6.1737

34. C. Kan, X. Zhu, G. Wang, Single-crystalline gold microplates: synthesis, characterization, and thermal stability. J. Phys. Chem. B 110(10), 4651-4656 (2006). doi:10.1021/jp054800d

35. J.E. Millstone, S. Park, K.L. Shuford, L. Qin, G.C. Schatz, C.A. Mirkin, Observation of a quadrupole plasmon mode for a colloidal solution of gold nanoprisms. J. Am. Chem. Soc. 127(15), 5312-5313 (2005). doi:10.1021/ja043245a

36. A.R. Siekkinen, J.M. McLellan, J. Chen, Y. Xia, Rapid synthesis of small silver nanocubes by mediating polyol reduction with a trace amount of sodium sulfide or sodium hydrosulfide. Chem. Phys. Lett. 432(4-6), 491-496 (2006). doi:10.1016/j.cplett.2006. 10.095

37. S. Kumar-Krishnan, E. Prokhorov, O. Arias de Fuentes, M. Ramírez, N. Bogdanchikova, I.C. Sanchez, J.D. Mota-Morales, G. Luna-Bárcenas, Temperature-induced au nanostructure synthesis in a nonaqueous deep-eutectic solvent for high performance electrocatalysis. J. Mater. Chem. A 3(31), 15869-15875 (2015). doi:10.1039/c5ta02606g

38. J. Zeng, X. Xia, M. Rycenga, P. Henneghan, Q. Li, Y. Xia, Successive deposition of silver on silver nanoplates: lateral versus vertical growth. Angew. Chem. Int. Ed. Engl. 50(1), 244-249 (2011). doi:10.1002/anie.201005549

39. J. Heo, Y.W. Lee, M. Kim, W.S. Yun, S.W. Han, Nanoparticle assembly on nanoplates. Chem. Commun. 15, 1981-1983 (2009). doi: $10.1039 / \mathrm{b} 821713 \mathrm{k}$

40. J.K. Daniels, G. Chumanov, Nanoparticle-mirror sandwich substrates for surface-enhanced Raman scattering. J. Phys. Chem. B 109(38), 17936-17942 (2005). doi:10.1021/jp053432a

41. K. Kim, J.K. Yoon, Raman scattering of 4-aminobenzenethiol sandwiched between $\mathrm{Ag} / \mathrm{Au}$ nanoparticle and macroscopically smooth au substrate. J. Phys. Chem. B 109(44), 20731-20736 (2005). doi:10.1021/jp052829b

42. J. Wu, Y. Xu, P. Xu, Z. Pan, S. Chen, Q. Shen, L. Zhan, Y. Zhang, W. Ni, Surface-enhanced Raman scattering from AgNPgraphene-AgNP sandwiched nanostructures. Nanoscale 7(41), 17529-17537 (2015). doi:10.1039/c5nr04500b

43. J. Tang, F.S. Ou, H.P. Kuo, M. Hu, W.F. Stickle, Z. Li, R.S. Williams, Silver-coated si nanograss as highly sensitive surfaceenhanced Raman spectroscopy substrates. Appl. Phy. A 96(4), 793-797 (2009). doi:10.1007/s00339-009-5305-0

44. S. Nie, Probing single molecules and single nanoparticles by surface-enhanced Raman scattering. Science 275(5303), 1102-1106 (1997). doi:10.1126/science.275.5303.1102

45. J. Jiang, K. Bosnick, M. Maillard, L. Brus, Single molecule Raman spectroscopy at the junctions of large ag nanocrystals. J. Phys. Chem. B 107(37), 9964-9972 (2003). doi:10.1021/ jp034632u

46. W. Ji, X. Xue, W. Ruan, C. Wang, N. Ji, L. Chen, Z. Li, W. Song, B. Zhao, J.R. Lombardi, Scanned chemical enhancement of surface-enhanced Raman scattering using a charge-transfer complex. Chem. Commun. 47(8), 2426-2428 (2011). doi:10.1039/ $\mathrm{c} 0 \mathrm{cc} 03697 \mathrm{~h}$

47. J. Cabalo, J.A. Guicheteau, S. Christesen, Toward understanding the influence of intermolecular interactions and molecular orientation on the chemical enhancement of sers. J. Phys. Chem. A 117(37), 9028-9038 (2013). doi:10.1021/jp403458k 